\title{
High sensitivity of ROSE-supported ERCP-guided brushing for biliary strictures
}

\section{다 $(9)$}

\section{Authors}

Livia Archibugi ${ }^{1}$, Alberto Mariani ${ }^{1}$, Biagio Ciambriello², Maria Chiara Petrone ${ }^{1}$, Gemma Rossi ${ }^{1}$, Sabrina Gloria Giulia Testoni ${ }^{1}$, Michele Carlucci ${ }^{3}$, Luca Aldrighetti ${ }^{4}$, Massimo Falconi ${ }^{5}$, Gianpaolo Balzano ${ }^{5}$, Claudio Doglioni ${ }^{6}$, Gabriele Capurso ${ }^{1}$, Paolo Giorgio Arcidiacono ${ }^{1}$

\section{Institutions}

1 Pancreato-Biliary Endoscopy and Endosonography Division, Pancreas Translational \& Clinical Research Center, Vita-Salute San Raffaele University, Milan, Italy, IRCCS San Raffaele Scientific Institute, Milan, Italy

2 Digestive Endoscopy AO Specialistica Dei Colli CTO, Naples, Italy

3 Department of General and Emergency Surgery, VitaSalute San Raffaele University Milan, Italy, IRCCS San Raffaele Scientific Institute, Milan, Italy

4 Hepatobiliary Surgery Division, Vita-Salute San Raffaele University Milan, Italy, IRCCS San Raffaele Scientific Institute, Milan, Italy

5 Pancreatic Surgery Unit, Pancreas Translational and Clinical Research Center, Vita-Salute San Raffaele University Milan, Italy, IRCCS San Raffaele Scientific Institute, Milan, Italy

6 Pathology Unit, Pancreas Translational and Clinical Research Center, Vita-Salute San Raffaele University Milan, Italy, IRCCS San Raffaele Scientific Institute, Milan, Italy

submitted 12.8 .2020

accepted after revision $\quad 21.10 .2020$

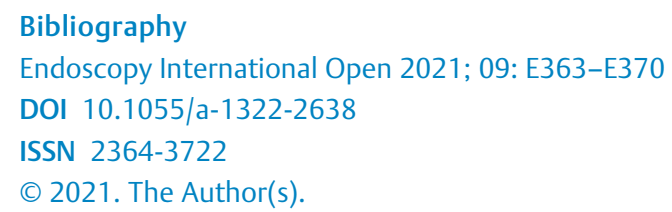
Commons Attribution-NonDerivative-NonCommercial License, permitting copying and reproduction so long as the original work is given appropriate credit. Contents may not be used for commecial purposes, or adapted, remixed, transformed or built upon. (https://creativecommons.org/licenses/by-nc-nd/4.0/)

Georg Thieme Verlag KG, Rüdigerstraße 14,

70469 Stuttgart, Germany

Corresponding author

Alberto Mariani, MD, Pancreato-Biliary Endoscopy and Endosonography Division, Pancreas Translational \& Clinical
Research Center, Via Olgettina 60, 20132, Milan, Italy Fax: +390226435609

mariani.alberto@hsr.it

\section{ABSTRACT}

Background and study aims Endoscopic retrograde cholangiopancreatography (ERCP) plays a major role in biliary strictures, with brushing being a cheap and fast method to acquire a cytological specimen, despite a sensitivity around $45 \%$. Rapid on-site evaluation (ROSE) is widely used for endoscopic ultrasound-acquired cytological specimen adequacy, improving its sensitivity and specificity. Nevertheless, no study has evaluated its role for ERCP-guided brushing. Our aim was to assess the diagnostic yield of ERCPguided brushing of biliary strictures when supported by ROSE.

Patients and methods This was a retrospective singlecenter study that included patients undergoing ERCP-guided brush cytology supported by ROSE for biliary strictures. Recorded data included patient clinical-radiological and ERCP features. Final diagnosis was determined after surgery, intraductal biopsy or adequate follow-up. The diagnostic yield was calculated and a subgroup analysis for factors associated with false-negative or true-positive results was performed.

Results Two hundred six patients were included, $57.3 \%$ males, median age 72 years, $77.2 \%$ having extrahepatic biliary strictures. Of the patients, $99 \%$ had an adequate sample at ROSE after a mean of 2.6 passages. The diagnostic yield was accuracy $83 \%$, sensitivity $74.6 \%$, and specificity $98 \%$, positive and negative predictive values $98 \%$ and $71 \%$ respectively, with an area under the curve of 0.86 . A diagnosis of cholangiocarcinoma was significantly more frequent among true-positive cases (68\% vs $46.8 \%$; $P=0.04$ ). Conclusions This is the first study evaluating the use of ROSE as support for ERCP-guided brushing of biliary strictures, with a sensitivity far higher than those reported for brushing alone and at least comparable to those of more expensive and invasive techniques. 


\section{Introduction}

Determination of the etiology of biliary strictures represents a significant challenge despite advances in endoscopic techniques. Taking into account both the aggressive behavior of pancreatobiliary neoplasms and the significant morbidity and mortality of pancreatobiliary surgery $[1,2]$, the correct distinction between a benign and malignant biliary stricture is of crucial importance. It has, indeed, been reported that two-thirds of indeterminate biliary strictures are malignant, but onefourth of all surgical resection specimens for this indication were reported as benign disease [3,4].

Many underlying diseases may cause biliary strictures, including malignancies (cholangiocarcinoma or pancreatic cancer in the majority of cases) and also benign disorders such as autoimmune processes (primary sclerosing cholangitis, immunoglobulin (lg)G4-related cholangitis, autoimmune pancreatitis), vascular injuries, and infectious diseases. Biliary strictures also can be the sequelae of iatrogenic or non-iatrogenic trauma (eg, post-cholecystectomy or long-standing choledocholithiasis) [5]. Most the time, distinguishing between these different etiologies is extremely cumbersome. Moreover, in case of locally advanced cancer, when the malignant etiology can be easily deducted, pathological proof is still necessary to plan chemotherapy.

Despite being considered a therapeutic technique, endoscopic retrograde cholangiopancreatography (ERCP) still has a prominent diagnostic role in patients with biliary strictures, especially when there is no evidence of mass lesion. Biliary stricture brushing is considered the safest, easiest, cheapest, and fastest way to acquire a cytological specimen from the biliary tree for diagnosing the etiology of a stricture, but despite a specificity of $99 \%$ to $100 \%$, the sensitivity is only around $45 \%$ [6]. Additional techniques can increase this sensitivity. Fluorescence in situ hybridization (FISH)-chromosomal aneuploidy on brushing specimen or on bile aspirate has a sensitivity of $34 \%$ to $52 \%$ [7], the use of optical coherence tomography (OCT) on the bile duct has a sensitivity of $53 \%$ [7,8], and combined use of cytology, FISH, and mutation profiling has been reported to increase sensitivity up to $69 \%$ [9].

Considering these figures, in the past few years, the field has moved toward acquisition of histological samples, with use of biopsy forceps, which have to be carefully guided to the site of the stricture. Therefore, cholangioscopes and cholangioscopydirected biopsy have been developed, achieving a sensitivity of $71.9 \%$ [10] despite heterogeneous results, with new evidence suggesting a lower diagnostic yield [11].

Rapid on-site evaluation (ROSE) of the cytological sample has been used for years for determination of adequacy of endoscopic ultrasonography-guided fine needle aspiration (EUSFNA) cytological specimens, improving its sensitivity and specificity [12]. Nevertheless, to the best of our knowledge, no studies have investigated the role of ROSE for ERCP-guided brushing.

The aim of our study, therefore, was to evaluate the diagnostic yield of the ERCP-guided brushing in determining the etiology of biliary strictures when supported by ROSE.

\section{Patients and methods \\ Study design and population}

A retrospective single-center study was conducted at IRCCS San Raffaele Hospital and enrolled patients undergoing ERCP for biliary strictures with a brushing technique supported by ROSE (IRB approval "Protocollo Registro ERCP 06/02/2014").

Data from all consecutive patients who had undergone ERCP with the brushing technique over an 11-year period (October 2008-October 2019) were retrieved from an electronic database in which information on the following variables was prospectively recorded: patient characteristics such as gender, age, presenting symptom, reports of computed tomography (CT) or magnetic resonance imaging (MRI) or EUS, whether the stricture was identified or not, site of the stricture, evidence of mass-lesion, indication for the ERCP procedure, procedure technical details including stricture location at ERCP cholangiography, brushing features such as number of passages to achieve adequacy, presence of a biliary stent (plastic or metallic) before the ERCP procedure, execution of bile duct biopsies during ERCP procedure, execution of follow-up through ERCP, use of confocal laser endomicroscopy (CLE), cholangioscopy, dilation of the stricture, execution of surgery, and final pathology. The same electronic database was used to retrieve data on patient follow-up.

The study population was selected to include patients undergoing ERCP with execution of brushing for the first time in the biliary tract, supported by ROSE.

Exclusion criteria were: 1. patients undergoing ERCP with brushing on the pancreatic duct; 2. patients undergoing brushing without the support of ROSE; 3. evidence of bile duct stricture due to liver hilum lymphadenopathies; 4 . absence of follow-up of at least 6 months or final histopathological diagnosis; and 5. patients undergoing a second session of ERCP with brushing (we considered only patients undergoing ERCP with brushing for the first time).

\section{Procedure description and specimen processing}

ERCP procedures were performed under deep sedation with intravenous infusion of propofol (Diprivan, Zeneca, Germany), using Pentax duodenoscopes (ED3470TK, ED34i10 T), by expert endoscopists who had performed over 200 procedures per year. Written informed consent was obtained from each included patient before the procedure.

During the procedure, biliary strictures were identified on cholangiography using lopamidol (Iopamiro, Bracco, Italy) contrast agent, and a cytopathological specimen was obtained using the Cytology Brush (Cook Medical, United States) or RX Cytology Brush (Boston Scientific, United States), with a nonstandardized number of brush passages defined arbitrarily until sample adequacy was obtained at ROSE. Specifically, after a first passage, the endoscopist would wait for the response regarding the adequacy to either perform additional passages until adequacy was obtained or move on to forceps biopsy or stent positioning. 
The brushing specimen was processed for ROSE by an onsite cytopathologist or cytotechnician, with the smears prepared immediately after obtaining the specimen by gently pressing and scraping the brush onto a clean glass slide to exfoliate the cells on it. At least two slides smears were prepared in each case, which were fixed in absolute alcohol and stained with a rapid 2-minute hematoxylin-eosin stain. Once the slides were prepared, they were examined by an onsite cytopathologist and real-time evaluation of the sample adequacy was performed. A sample was considered adequate based on whether there was enough material representative of the site of sampling, independent of whether it was deemed benign or malignant and irrespective of clinical suspicion. A diagnostic category was provided, depending upon whether the nature of the cells was benign (when the cytologic specimen did not reveal malignancy), suspicious, or malignant. The diagnosis was based on classic cytologic criteria, i.e nuclear shape and dimension, hyperchromasia, high nuclear-to-cytoplasmic ratio, cyto-architectural abnormalities, and necrotic background. The onsite cytopathologist was not blinded to patient clinical and radiological history.

A forceps biopsy could be performed in cases of adequate location and conformation of the stricture, based on endoscopist preference and always after at least a first brush passage.

Subsequent ERCP sessions were performed on patients with indeterminate diagnosis both to repeat the brushing, perform biopsies or study the stricture with the cholangioscope, or to exchange the plastic stent in place.

\section{Definition of the gold-standard and other definitions}

A case was considered as "true positive" when, after a cytologic diagnosis suspicious for malignancy or of malignancy on ROSE, the patient was found to have a malignant stricture. The definition of malignancy was based on a final histopathological diagnosis on a surgical sample from patients who had undergone surgery and evidence of malignancy on histopathological evaluation of the specimen from forceps biopsy or in patients who did not undergo surgery, on an EUS-FNA sample performed on the stricture or evidence of progression of the disease after at least 6 months of follow-up (onset of liver or peritoneal metastases or growth of the mass lesion with evidence of vascular infiltration if not present at baseline).

A case was considered as "true negative" when, after a benign cytologic diagnosis on ROSE, the patient was found to have a benign stricture as defined by a final histopathological diagnosis based on a surgical sample in patients who underwent surgery and evidence of stability or disappearance of the stricture after at least 6 months of follow-up in patients who did not undergo surgery.

In cases in which baseline CT scan, MRI or EUS revealed the presence of a solid mass-forming stricture of the bile duct (arising either from the pancreas or from any tract of the bile duct) and not just a thickening of bile duct walls, the case was considered as being a "mass-forming" disease.

\section{Statistical analysis}

Descriptive statistics were calculated as means and standard deviation (SD) for normally distributed continuous variables, as means and SD and median and interquartile range (IQR) for continuous variables with skewed distribution, and as numbers and percentages for categorical variables.

A paired Student's $t$-test was employed for comparison of normally distributed continuous variables, a Mann-Whitney $U$ test was used for continuous variables with skewed distribution, and Fisher's exact test was employed for the comparison of categorical variables.

Accuracy, sensitivity, specificity, positive predictive value (PPV) and negative predictive value (NPV) for ERCP-guided brushing plus ROSE diagnosis were calculated. A receiver operating characteristic (ROC) curve was then plotted and area under the curve $(A \cup C)$ calculated.

After evaluation of the diagnostic yield of ROSE, characteristics of true-positive and false-negative cases were compared. All calculations were performed using MedCalc version 13 (MedCalc Software, Belgium). $P<0.05$ was considered statistically significant.

The "Standards for Reporting Diagnostic accuracy studies" (STARD) and "STrengthening the Reporting of OBservational studies in Epidemiology" (STROBE) checklists were checked for items that should be included in the report.

\section{Results}

\section{Patients and biliary stricture characteristics}

Of 5,929 ERCP procedures performed between October 2008 and October 2019, 5,640 were excluded, as reported in - Fig. 1. The final number of patients included in the analysis was 206, 118 of whom were male (57.3\%) with a median age at diagnosis of 72 years ( $\vee$ Table 1 ).

The majority of patients presented with jaundice (68\%), with a stricture in the common bile duct (77.2\%) and a mass-forming lesion in $21 \%$ of cases.

\section{Diagnostic yield of ERCP-guided brushing supported by ROSE in establishing the etiology of the biliary stricture}

Overall, according to the aforementioned criteria, 126 patients (61.2\%) were deemed to have a malignant stricture and 80 patients (38.8\%) a benign stricture. The outcome (gold-standard) was defined in 67 cases $(32.5 \%)$ based on surgical resection (whether benign or malignant at histopathological examination) in $24(11.7 \%)$ and 15 (7.3\%) cases based on the malignant result of intraductal biopsy and EUS-FNA, respectively. In the remaining cases, the outcome was defined based on evidence of progressive disease or stable/remitted disease at a mean follow-up time of 40.8 months (median 26.5 months) ( $\triangleright$ Table 2 ).

Ninety-eight percent of patients (203 of 206) had an adequate sample at ROSE ( $>$ Fig. 2 ) after a mean number of passages of $2.6( \pm 0.6)$ (range $1-4)$ ( $>$ Table 1 ); the $2 \%$ (3 cases) with non-adequate samples underwent a mean number of brush passages of three. 
ERCPs performed between Oct 2008 - Oct 2019

5929

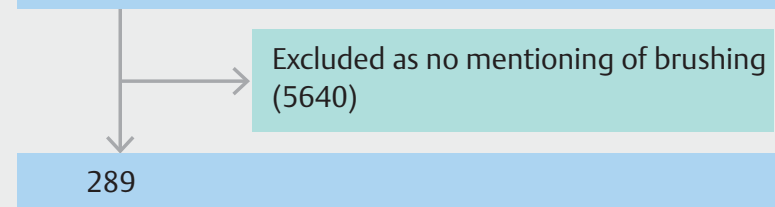

Excluded as meeting exclusion criteria (83):

- 30 pts with brushing not performed on the biliary tract

- 3 with evidence of lymphadenopathies compressing the bile duct

- 50 pts with no adequate follow-up or final histopathological diagnosis available

\section{ERCP-guided brushing supported by ROSE}

> Fig. 1 Flowchart of patient selection for ERCP-guided brushing supported by ROSE.

The diagnostic accuracy of ERCP-guided brushing with ROSE in determining the correct etiology of biliary strictures was $83.5 \%$. Sensitivity and specificity were $74.6 \%$ and $97.5 \%$, respectively ( $\triangleright$ Table 3 ), with an AUC of 0.86 ( $\triangleright$ Fig.3). PPV and NPV were $97.9 \%$ and $70.9 \%$, respectively. Of the nine benign strictures treated with surgical resection, four had a postflogistic stricture ( 2 of them undergoing resection for stricture leading to multiple episodes of cholangitis that did not respond to endoscopic treatments) and five had IgG4-related cholangitis.

Of the 206 patients, three underwent cholangioscopy, with a visual report of a benign stricture in all cases ( 2 true-negative, 1 false-negative).

Accuracy for $\leq 2$ brush passages was $90 \%$ and for $\geq 3$ passages, it was $82 \%$.

\section{Analysis of factors associated with true-positive versus false-negative results and number of passages in performance of ERCP-guided brushing supported by ROSE}

To identify possible factors associated with true-positive/falsenegative results, a subanalysis comparing the two groups was performed. As shown in $>$ Table 4 , false-negative cases were affected less frequently by cholangiocarcinoma of the bile duct ( $46.8 \%$ vs $68 \% ; P=0.04)$ and more often by pancreatic cancer ( $28.1 \%$ vs $14.8 \% ; P=0.01)$ and gallbladder cancer $(18.7 \%$ vs $5.3 \% ; P=0.03)$. No significant differences were found in terms of age, gender, presenting symptoms, stricture location, massforming disease, biliary stent presence before the brushing (despite being double among the false negative cases), or mean number of brush passages.
- Table 1 Patient and biliary stricture characteristics.

\begin{tabular}{|c|c|}
\hline & $\begin{array}{l}\text { Total patients } \\
\text { enrolled } \\
(n=206)\end{array}$ \\
\hline \multicolumn{2}{|l|}{ Age, years } \\
\hline - Mean ( \pm SD) & $69.2( \pm 12.9)$ \\
\hline - Median (IQR) & $72(60-78)$ \\
\hline Sex, male, n (\%) & $118(57.3 \%)$ \\
\hline \multicolumn{2}{|l|}{ Dominant presenting symptom/sign } \\
\hline - Jaundice & $140(68 \%)$ \\
\hline - Abdominal pain & $24(11.6 \%)$ \\
\hline - Incidental finding of increased liver tests & $6(2.9 \%)$ \\
\hline - Incidental finding of dilated bile duct & $6(2.9 \%)$ \\
\hline - Biliary stones (either first diagnosis or follow-up) & $5(2.4 \%)$ \\
\hline - Post-ampullectomy follow-up & $3(1.4 \%)$ \\
\hline - Acute pancreatitis & $2(0.9 \%)$ \\
\hline - Other & $20(9.7 \%)$ \\
\hline \multicolumn{2}{|l|}{ Location of the stricture } \\
\hline - Peri-hilar or intrahepatic & $37(18 \%)$ \\
\hline - Distal (stricture of the CBD) & $159(77.2 \%)$ \\
\hline - Proximal third & $25(12.1 \%)$ \\
\hline - Middle third & $33(16 \%)$ \\
\hline - Distal third & $70(34 \%)$ \\
\hline - Diffuse/multifocal ${ }^{1}$ & $31(15.1 \%)$ \\
\hline - Diffuse/multifocal ${ }^{2}$ & $10(4.8 \%)$ \\
\hline \multicolumn{2}{|l|}{ Final clinical/histological diagnosis of the strictures } \\
\hline - Cholangiocarcinoma of the bile duct & $79(38.4 \%)$ \\
\hline - Gallbladder Cancer & $11(5.3 \%)$ \\
\hline - Pancreatic Cancer & $23(11.2 \%)$ \\
\hline - Other cancers ${ }^{3}$ & $13(6.3 \%)$ \\
\hline - IgG4-related disease & $5(2.4 \%)$ \\
\hline - Post-cholecystectomy or flogistic stricture & $75(36.4 \%)$ \\
\hline Mass forming & $44(21.4 \%)$ \\
\hline Arising on Primary Sclerosing Cholangitis & $4(1.9 \%)$ \\
\hline Presenting with stent at ERCP with brushing session & $40(19.4 \%)$ \\
\hline - Plastic stent & $36(17.5 \%)$ \\
\hline - Metal stent & $4(1.9 \%)$ \\
\hline Number of brushing passages, mean $( \pm S D)$ & $2.6( \pm 0.6)$ \\
\hline
\end{tabular}

SD, standard deviation; IQR, interquartile range; CBD, common bile duct; $\mathrm{IgG}$, immunoglobulin G; ERCP, endoscopic retrograde cholangiopancreatography.

${ }^{1}$ Affecting more than one third of the CBD.

${ }^{2}$ Affecting both the CBD and the peri-hilar or peri-hilar and intrahepatic ducts.

${ }^{3}$ Hepatocellular carcinoma, neuroendocrine neoplasia, ampullary carcino$\mathrm{ma}$, and intraductal papillary mucinous neoplasm of the biliary tract. 


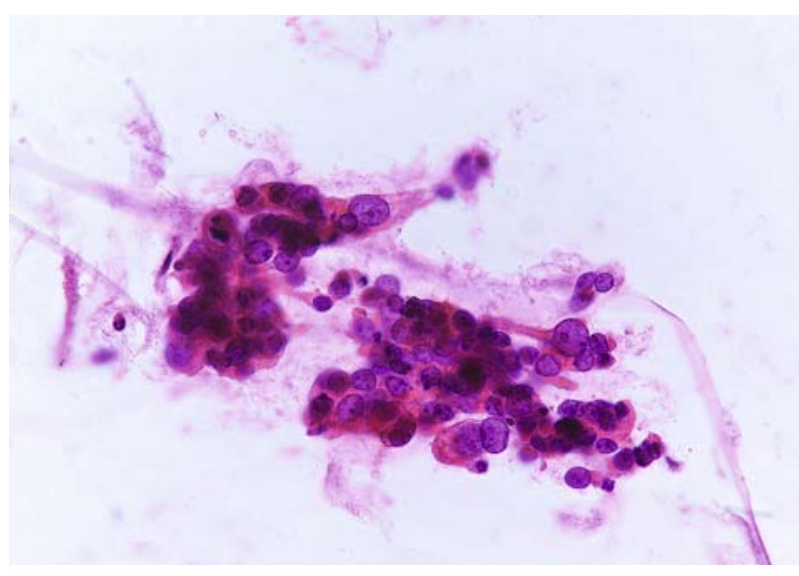

- Fig. 2 Brushing sample adequate for malignancy, with enlarged hyperchromatic nuclei, high nuclear-to-cytoplasmic ratio, and necrotic background.

- Table2 Assessment of outcome through surgery, biopsy, EUS-FNA and follow-up.

\begin{tabular}{|l|c|}
\hline Surgical resection & $67(32.5 \%)^{1}$ \\
\hline - Benign & $9(4.4 \%)$ \\
\hline - Malignant & $58(28.2 \%)$ \\
\hline Intraductal biopsy & $44(21.4 \%)$ \\
\hline - Benign & $20(9.7 \%)$ \\
\hline - Malignant & $24(11.7 \%)^{1}$ \\
\hline EUS-FNA & $29(14.1 \%)$ \\
\hline - Benign & $9(4.4 \%)$ \\
\hline - Malignant & $15(7.3 \%)^{1}$ \\
\hline - Inadequate & $5(2.4 \%)$ \\
\hline $\begin{array}{l}\text { Follow-up length of the remaining patients } \\
\text { (months) }\end{array}$ & $100(48.5 \%)^{1}$ \\
\hline - Mean \pm SD & $40.8 \pm 33.4$ \\
\hline - Median (IQR) & $26.5(15.5-64.8)$ \\
\hline $\begin{array}{l}\text { - Evidence of disease progression (inter- } \\
\text { preted as malignancy) }\end{array}$ & $29(14.1 \%)$ \\
\hline $\begin{array}{l}\text { - Evidence of stricture stability/resolution } \\
\text { (interpreted as benign disease) }\end{array}$ & $71(34.4 \%)$ \\
\hline $\begin{array}{l}\text { - } \\
\text { - }\end{array}$ & \\
\hline - & \\
\hline
\end{tabular}

EUS-FNA, endoscopic ultrasound-guided fine needle aspiration; SD, standard deviation; IQR, interquartile range.

1 Used as gold-standard

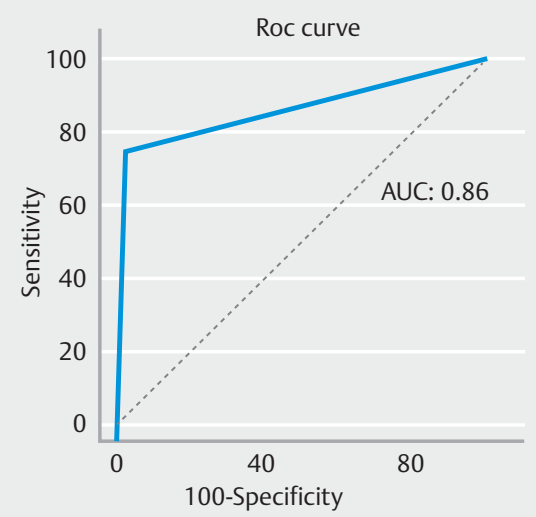

- Fig. 3 Receiver operating characteristic (ROC) curves and area under the curve (AUC) for the accuracy of ERCP-guided brushing supported by ROSE in establishing biliary stricture etiology. The $\mathrm{AUC}$ is 0.86 .

Patients who underwent $\leq 2$ or $\geq 3$ brushing passages were not statistically different in terms of having a biliary stent in place at first procedure ( $100 \%$ vs $94 \%$ ), having mass forming disease (30\% vs $20 \%$ ), or location of stenosis ( $70 \%$ vs $70.1 \%$ with an extrahepatic stenosis, $20 \%$ vs $23.5 \%$ with hilary stricture and $10 \%$ vs $6.4 \%$ with an extrahepatic stricture).

\section{Discussion}

Correctly diagnosing the etiology of a biliary stricture remains challenging. ERCP-guided brushing is the cheapest and easiest technique, but its sensitivity is disappointing at around $45 \%$ [6]. Therefore, the use of more expensive, complex, and invasive techniques, such as cholangioscopy-guided biopsy with a sensitivity around $72 \%$ [10], are increasingly being suggested and performed.

ROSE has been used for years to increase the diagnostic yield of EUS-FNA adequacy, but its role has never been explored in the context of ERCP-guided brushing, where it may also assist in immediate decision-making.

In the present study, the use of ROSE as support for ERCPguided biliary brushing was evaluated for the first time in a cohort of 206 patients, resulting in a sensitivity of $74.6 \%$ and a final AUC of 0.86 . The mean number of brush passages to achieve adequacy was 2.6 .

These data seem very promising compared to the majority of techniques currently performed, such as the addition of fluoroscopy-guided biopsy, with a sensitivity of $67.9 \%$ [13],

- Table 3 Diagnostic yield of ERCP-guided brushing supported by ROSE in establishing the etiology of biliary strictures.

\begin{tabular}{|c|c|c|c|c|c|c|c|c|c|c|}
\hline TP & TN & FP & FN & Accuracy & Sensitivity & Specificity & PPV & NPV & + +LR \\
\hline 94 & 78 & 2 & 32 & $83.5 \%$ & $74.6 \%$ & $97.5 \%$ & $97.9 \%$ & $70.9 \%$ & 30 \\
\hline
\end{tabular}

TP, true positive; TN, true negative; FP, false positive; FN, false negative; PPV, positive predictive value; NPV, negative predictive value; +LR, positive likelihood ratio; $-L R$, negative likelihood ratio. 
- Table 4 Comparison of patient and lesion variables in true-positive and false-negative cases.

\begin{tabular}{|c|c|c|c|}
\hline & $\begin{array}{l}\text { True-positive } \\
(\mathrm{n}=94)\end{array}$ & $\begin{array}{l}\text { False-negative } \\
(n=32)\end{array}$ & $P$ value \\
\hline Age (years), median (IQR) & $72(62.5-80)$ & $73(59.2-75.5)$ & 0.27 \\
\hline $\operatorname{Sex}(M)$ & $51(54.2 \%)$ & $15(46.9 \%)$ & 0.5 \\
\hline Presenting with jaundice & 77 (81.9\%) & $24(75 \%)$ & 0.4 \\
\hline \multicolumn{4}{|l|}{ Location of the stricture } \\
\hline - Peri-hilar or intrahepatic & $24(25.5 \%)$ & $7(21.9 \%)$ & 0.8 \\
\hline - Distal (stricture of the CBD) & $62(66 \%)$ & $23(71.9 \%)$ & 0.6 \\
\hline - Diffuse/multifocal & $8(8.5 \%)$ & $2(6.3 \%)$ & 1 \\
\hline Mass forming & $29(30.9 \%)$ & $12(37.5 \%)$ & 0.5 \\
\hline Presenting with plastic/metal stent & $10(10.6 \%)$ & $7(21.9 \%)$ & 0.13 \\
\hline Brush passages, mean $\pm \mathrm{SD}$ & $2.5 \pm 0.6$ & $2.7 \pm 0.6$ & 0.11 \\
\hline \multicolumn{4}{|l|}{ Final malignant etiology } \\
\hline - Cholangiocarcinoma of the bile duct & $64(68 \%)$ & $15(46.8 \%)$ & 0.04 \\
\hline - At surgical specimen & $26(27.6 \%)$ & $12(37.5 \%)$ & \\
\hline - Based on radiologic and EUS findings & $38(40.4 \%)$ & $3(9.3 \%)$ & \\
\hline - Gallbladder cancer & $5(5.3 \%)$ & $6(18.7 \%)$ & 0.03 \\
\hline - At surgical specimen & 0 & $1(3.1 \%)$ & \\
\hline - Based on radiologic and EUS findings & $5(5.3 \%)$ & $5(15.6 \%)$ & \\
\hline - Pancreatic Cancer & $14(14.8 \%)$ & $9(28.1 \%)$ & 0.01 \\
\hline - At surgical specimen & $5(5.3 \%)$ & $7(21.9 \%)$ & \\
\hline - Based on radiologic and EUS findings & $9(9.5 \%)$ & $2(6.2 \%)$ & \\
\hline - Other malignant etiologies/unclear etiology & $11(11.7 \%)$ & $2(6.2 \%)$ & 0.51 \\
\hline
\end{tabular}

FISH, with a sensitivity of $44 \%$ [9], use of digital single-operator cholangioscopy, with recent reports of a sensitivity for visual interpretation ranging between $64 \%$ [11] and $89.1 \%$ [14], and for bile duct sampling under cholangioscopic guidance, with sensitivity ranging between 15\% [11] and 69.8\% [14].

Data are extremely limited on application of ROSE in cases other than EUS-FNA. Ali et al. [15] retrospectively investigated its role in touch imprint cytology (TIC) of biopsies of intestinal luminal or pancreatobiliary lesions taken during ERCP with cholangioscopy. In the 121 pancreatobiliary lesions, the resulting sensitivity was $97 \%$. However, this high yield has to be viewed with caution, as the outcome definition used as gold standard was based only on the final histopathological result of the biopsy. In the present study, the outcomes used as goldstandard were not related to the final report of the brushing itself, but we considered either the final histopathological diagnosis based on other techniques (surgical resection, intraductal biopsies, EUS-FNA) or a follow-up that was more than 3 years on average. Indeed, in the decision-making process, the role of ROSE is far more relevant during EUS-FNA because of the higher risk of complications associated with the higher number of pas- sages compared to the remarkable safety of ERCP-guided brushing. Nevertheless, because this is the first study on the topic, identification of the mean number of passages needed to achieve an adequacy can help guide centers in which the ROSE is not available and it also can support endoscopists performing ERCP in decision-making about stent positioning.

As with any method, the definition of the proper target population for applying this technique is crucial and can help guide physicians. Some malignant diseases can lead to biliary strictures due to extraluminal compression, which are therefore expected to lead to false-negative results from both brushing and biopsy specimens. These diseases include pancreatic cancer, hepatocellular carcinoma or hepatic metastases, gallbladder cancer, and metastatic adenopathies of the liver hilum [5]. For this reason, in the present study, we excluded patients with metastatic adenopathies of the liver hilum and performed a subgroup analysis of false-negative and true-positive cases to investigate the possible role of the specific malignancy type and of other factors, such as the presence of a biliary stent before the procedure [16]. In this subgroup analysis, false-negative cases more often had a final diagnosis of gallbladder or pancre- 
atic cancer, and less often cholangiocarcinoma. In cholangiocarcinoma, the peculiar growth of the tumour (periductal infiltrating or intraductal growing) can make it more likely that with brushing, an adequate sample of malignant tissue can be collected in cases with intraductal growing; the risk of false-negative results is higher for periductal infiltrating cases [17]. Nevertheless, the type of growth of cholangiocarcinoma cannot be hypothesized based on imaging findings and can only be deduced from a surgical specimen. Despite being statistically insignificant, the presence of a biliary stent before brushing was more prevalent in false-negative cases compared to truepositive cases ( $22 \%$ vs $10 \%$ ), suggesting that it might reduce the sensitivity of brushing. Age, gender, stricture location, mass-forming disease or number of brush passages did not differ between the two groups. In addition, a slightly higher number of passages was not associated with higher accuracy, suggesting that the tumor growth pattern (intraductal vs periductal) is a more relevant factor.

This study has some strengths. It was the first study evaluating the use of ROSE for ERCP-guided brushing; the aim, inclusion, and exclusion criteria were clearly defined; it had a large sample size; and the diagnostic yield was calculated with strictly defined gold standards and with a long follow-up.

However, there are limitations such as the lack of a control group, the retrospective part of the study, and the heterogeneous gold-standard, as not all patients underwent surgical resection. Furthermore, ROSE often is not available; nevertheless, a 2016 survey demonstrated that $65 \%$ of EUS centers have ROSE, $98 \%$ in the United States and $50 \%$ in Europe and Asia, respectively [18]. In addition, the expertise of the pathologists in a high-volume referral center may not be replicated in nontertiary centers, thus the present results may not be widely applicable. In fact, the accuracy of brushing is highly dependent upon processing and evaluation of samples, which in turn differs among different centers. Another possible limitation is selection bias, meaning that this cohort of patients undergoing biliary brushing at our tertiary referral center is not fully representable of other cohorts of patients with biliary strictures. Indeed, in our center, the majority of patients would undergo EUS-FNA first, if it is possible to sampling the lesion. Therefore, diagnosis would be achieved in a high percentage of patients before ERCP and they would not undergo sampling with brushing. This also could have led to selection of more complex cases in which EUS-FNA had failed (as in — Table 2, 14\% of patients already underwent EUS-FNA) and, therefore, performance of ROSE for ERCP-guided brushing may be underestimated. It would be interesting to investigate whether ROSE for ERCPguided brushing has even higher accuracy in centers where EUS-FNA is less readily available or is performed by operators with less expertise. Finally, bile intraductal aspiration for collection of additional cytological material was not performed; use of this technique before or after brushing could further increase the diagnostic yield $[16,19-21]$ and may deserve further investigation.

Given such limitations, the present results should be interpreted with caution and acknowledgment that replication of them is needed. Nevertheless, we believe that further prospec- tive investigation is warranted of ERCP-guided brushing supported by ROSE as a first approach to biliary strictures. The present results may, indeed, suggest that an effort in training the Pathology Unit [22] could be far more cost-effective than use of advanced technology. A recent study by Deprez et al. [23] investigated the economic impact of cholangioscopy for biliary strictures, reporting a unit cost for ERCP with brushing of $1,699 €$ and $3,946 €$ for cholangioscopy-guided biopsies. The authors concluded that because in the longer term upfront use of cholangioscopy could lead to a reduction in need for repeat procedures, its upfront use might eventually lead to significant cost reduction. Notably, in a recent study, Singhi et al. [24] evaluated use of a 28-gene next generation sequencing (NGS) panel on ERCP-guided acquired pathological specimens from biliary strictures to evaluate its effectiveness in improving the diagnostic sensitivity of commonly performed techniques. The use of this panel on brushing specimens helped increase the sensitivity from $35 \%$ to $77 \%$, data comparable to ours, albeit with the higher cost of NGS. The use of ROSE to guide brushing during a first session of ERCP for biliary strictures prolongs the procedure by a few minutes compared to a standard ERCP with brushing and requires an on-site cytopathologist, resulting in additional costs. However, it is likely that these costs are well balanced by avoiding that of repeated ERCP with cholangioscopy and/or molecular diagnostics. These hypotheses need to be confirmed with prospective head-to-head trials that include cost-effectiveness analyses.

\section{Conclusion}

In conclusion, this was the first study on the role and diagnostic yield of ROSE for ERCP-guided brushing of biliary strictures, with findings of very high sensitivity, considering the safety, cheapness, rapidity, and simplicity of the technique. Further prospective and controlled studies are recommended to determine whether ERCP-guided brushing with the aid of ROSE can be considered as that first step for diagnosis of the etiology of biliary strictures and reduce the need for multiple ERCP sessions and use expensive adjunctive techniques.

\section{Competing interests}

The authors declare that they have no conflict of interest.

\section{References}

[1] Roos E, Strijker M, Franken LC et al. Comparison of short- and longterm outcomes between anatomical subtypes of resected biliary tract cancer in a Western high-volume center. HPB (Oxford) 2020; 22: 405414

[2] Balzano G, Zerbi A, Capretti G et al. Effect of hospital volume on outcome of pancreaticoduodenectomy in Italy. Br J Surg 2008; 95: 357362

[3] Bowlus C, Olson K, Gershwin E. Evaluation of indeterminate biliary strictures. Nat Rev Gastroenterol Hepatol 2016; 13: 28-37 
[4] Tummala P, Munigala S, Eloubeidi MA et al. Patients with obstructive jaundice and biliary stricture \pm mass lesion on imaging: prevalence of malignancy and potential role of EUS-FNA. J Clin Gastroenterol 2013; 47: 532-537

[5] Novikov A, Kowalski TE, Loren DE. Practical Management of Indeterminate Biliary Strictures. Gastrointest Endosc Clin N Am 2019; 29: 205-214

[6] Navaneethan U, Njei B, Lourdusamy V et al. Comparative effectiveness of biliary brush cytology and intraductal biopsy for detection of malignant biliary strictures: a systematic review and meta-analysis. Gastrointest Endosc 2015; 81: 168-176

[7] Burnett AS, Calvert T], Chokshi RJ. Sensitivity of endoscopic retrograde cholangiopancreatography standard cytology: 10-y review of the literature. J Surg Res 2013; 184: 304-311

[8] Testoni PA, Mangiavillano B. Optical coherence tomography in detection of dysplasia and cancer of the gastrointestinal tract and biliopancreatic ductal system. World J Gastroenterol 2008; 14: 64446452

[9] Kushnir VM, Mullady DK, Das K et al. The diagnostic yield of malignancy comparing cytology, FISH, and molecular analysis of cell free cytology brush supernatant in patients with biliary strictures undergoing endoscopic retrograde cholangiography (ERC): a prospective study. J Clin Gastroenterol 2019; 53: 686-692

[10] Badshah MB, Vanar V, Kandula M et al. Peroral cholangioscopy with cholangioscopy-directed biopsies in the diagnosis of biliary malignancies: a systemic review and meta-analysis. Eur J Gastroenterol Hepatol 2019; 31: 935-940

[11] de Vries AB, van der Heide F, Ter Steege RWF et al. Limited diagnostic accuracy and clinical impact of single-operator peroral cholangioscopy for indeterminate biliary strictures. Endoscopy 2020; 52: 107-114

[12] Iglesias-Garcia J, Lariño-Noia J, Abdulkader I et al. Rapid on-site evaluation of endoscopic-ultrasound-guided fine-needle aspiration diagnosis of pancreatic masses. World J Gastroenterol 2014; 20: 94519457

[13] Han S, Tatman P, Mehrotra S et al. Combination of ERCP-Based Modalities Increases Diagnostic Yield for Biliary Strictures. Dig Dis Sci 2020: doi:10.1007/s10620-020-06335-x

[14] Jang S, Stevens T, Kou L et al. Efficacy of digital single-operator cholangioscopy and factors affecting its accuracy in the evaluation of indeterminate biliary stricture. Gastrointest Endosc 2020; 91: 385393.e1
[15] Ali S, Hawes RH, Kadkhodayan K et al. Utility of rapid onsite evaluation of touch imprint cytology from endoscopic and cholangioscopic forceps biopsy sampling (with video). Gastrointest Endosc 2019; 89: 340-344

[16] Roth GS, Bichard P, Fior-Gozlan M et al. Performance of bile aspiration plus brushing to diagnose malignant biliary strictures during endoscopic retrograde cholangiopancreatography. Endosc Int Open 2016; 4: E997-E1003

[17] Kendall T, Verheij ], Gaudio E et al. Anatomical, histomorphological and molecular classification of cholangiocarcinoma. Liver Int 2019; 39: 7-18

[18] van Riet PA, Cahen DL, Poley JW et al. Mapping international practice patterns in EUS-guided tissue sampling: outcome of a global survey. Endosc Int Open 2016; 4: E360-E370

[19] Kobayashi M, Ryozawa S, Araki R et al. Investigation of Factors Affecting the Sensitivity of Bile Duct Brush Cytology. Intern Med 2019; 58: 329-335

[20] Fior-Gozlan M, Giovannini D, Rabeyrin M et al. Monocentric study of bile aspiration associated with biliary brushing performed during endoscopic retrograde cholangiopancreatography in 239 patients with symptomatic biliary stricture. Cancer Cytopathol 2016; 124: 330-339

[21] Curcio G, Granata A, Barresi L et al. Bile intraductal aspiration (BIDA): a fast method for bile collection. Endoscopy 2012; 44: E230-E231

[22] Petrone MC, Arcidiacono PG, Carrara S et al. Does cytotechnician training influence the accuracy of EUS-guided fine-needle aspiration of pancreatic masses? Dig Liver Dis 2012; 44: 311-314

[23] Deprez PH, Garces Duran R, Moreels T et al. The economic impact of using single-operator cholangioscopy for the treatment of difficult bile duct stones and diagnosis of indeterminate bile duct strictures. Endoscopy 2018; 50: 109-118

[24] Singhi AD, Nikiforova MN, Chennat J et al. Integrating next-generation sequencing to endoscopic retrograde cholangiopancreatography (ERCP)-obtained biliary specimens improves the detection and management of patients with malignant bile duct strictures. Gut 2020; 69: 52-61 